\begin{tabular}{|c|l|}
\hline Title & Electron transport in a gold nanoparticle assembly structure stabilized by a phy sisorbed porphyrin derivative \\
\hline Author(s) & Noda, Y uki; Noro, Shin-ichiro; A kutagawa, Tomoyuki; Nakamura, Takay oshi \\
\hline Citation & $\begin{array}{l}\text { Physical Review B, 82(20), 205420 } \\
\text { https://doi.org/_0.1103/PhysRevB.82.205420 }\end{array}$ \\
\hline Issue Date & 2010-11-15 \\
\hline Doc URL & http://hdl.handle.net/2115/44428 \\
\hline Rights & ○2010 The A merican Physical Society \\
\hline Type & article \\
\hline File Information & PRB82-20_205420.pdf \\
\hline
\end{tabular}

Instructions for use 


\title{
Electron transport in a gold nanoparticle assembly structure stabilized by a physisorbed porphyrin derivative
}

\author{
Yuki Noda, ${ }^{1}$ Shin-ichiro Noro, ${ }^{1,2}$ Tomoyuki Akutagawa, ${ }^{3}$ and Takayoshi Nakamura ${ }^{1,2}$ \\ ${ }^{1}$ Graduate School of Environmental Science, Hokkaido University, N10W5, Kita-ku, Sapporo 060-0810, Japan \\ ${ }^{2}$ Research Institute for Electronic Science, Hokkaido University, N20W10, Kita-ku, Sapporo 001-0020, Japan \\ ${ }^{3}$ Institute of Multidisciplinary Research for Advanced Materials, Tohoku University, 1-1 Katahira, 2-Chome, Sendai 980-8577, Japan
}

(Received 1 July 2010; revised manuscript received 1 October 2010; published 12 November 2010)

Gold nanoparticles stabilized by meso-5,10,15,20-tetrakis(2-thienyl)porphyrin (2T) via physisorption (2T-AuNP) were synthesized, and the electronic transport of assemblies of these films was studied. The adsorption mechanism of $2 \mathrm{~T}$ on gold nanoparticles was examined using UV-vis-NIR, IR, Raman, and ${ }^{1} \mathrm{H}-\mathrm{NMR}$ spectroscopy, which showed no evidence of any covalent bonding between $2 \mathrm{~T}$ and the gold nanoparticles. In temperature-dependent resistivity measurements, a crossover from thermally assisted hopping to EfrosShklovskii-type variable-range hopping (ES-VRH) was observed around $50 \mathrm{~K}$ on decreasing the temperature. At higher temperatures, the 2T-AuNP assembly structure followed an Arrhenius plot $\left(E_{A}=15 \mathrm{meV}\right)$ with ohmic $I-V$ characteristics at each measurement point. On the other hand, the activation energy at lower temperatures decreased nonlinearly in a $T^{-1}$ plot, and the logarithm of the resistance obeyed a $T^{-1 / 2}$ law, corresponding to an ES-VRH mechanism, which is predicted for disordered materials as a variable-range hopping mechanism influenced by strong Coulomb interactions. ES-VRH behavior has been observed previously in saturated molecule/gold nanoparticle assemblies and was confirmed in our 2T-AuNP assembly. Electronically active conjugated molecules were successfully incorporated between the nanoparticles, keeping the electronic structure of the gold nanoparticle and $2 \mathrm{~T}$ moieties isolated from each other.

DOI: 10.1103/PhysRevB.82.205420

PACS number(s): 72.80.Tm

\section{INTRODUCTION}

Gold nanoparticle assemblies have attracted much attention because they are predicted to act as building blocks for new electronic devices. A number of studies have been carried out to connect gold nanoparticles with organic molecules, and the electron transport properties of the resulting network structures have been evaluated. For example, gold nanoparticle saturated alkane assemblies show metalinsulator transitions, depending on the strength of the internanoparticle coupling, which is regulated by the internanoparticle separation distance. ${ }^{1}$ Normal and inverse photoconductivity has been observed by irradiation using visible light close to the surface-plasmon resonance of the gold nanoparticles. ${ }^{2}$ Spin-polarized molecular wires connecting the gold nanoparticles showed negative magnetoresistance behavior. ${ }^{3}$ The electron transport properties of the assembled gold nanoparticles are strongly dependent on the assembly structure, as well as on the organic molecules connecting the nanoparticles. A wide range of conduction models has been proposed so far.

Protecting ligands, such as sulfur, phosphine, citrate, amine, carboxylate, and isocyanide groups have been used to stabilize the gold nanoparticles. Among these, sulfurstabilized gold nanoparticles have been widely applied, because they allow for the facile synthesis of thermally stable particles with a narrow size dispersion, and the incorporation of a conjugated molecule is possible by utilizing the strong covalent bonds between the $\mathrm{Au}$ and $\mathrm{S}$ atoms. ${ }^{4}$ Investigations into the electron transport properties of gold nanoparticle assemblies have been mainly carried out on Au-S-stabilized nanoparticles. For example, Torma et al. ${ }^{5}$ have reported on the influence of chemical bonds on the activation energy us- ing covalent or noncovalently linked gold nanoparticle assemblies employing saturated alkyl dithiolate or thiolate ligands, respectively. For noncovalently linked nanoparticles, the activation energy shows a linear relationship with internanoparticle distance, with a minor dependence on the chemical structure of the linker molecules. On the other hand, covalently linked nanoparticles show a nonlinear relationship with the internanoparticle distance. The latter results suggest that the molecular orbitals of the linking molecules play an important role in the electron transport properties of the gold nanoparticle assemblies. Wessels et $a l .{ }^{6}$ have studied the conductivity of gold nanoparticle assemblies from the viewpoint of the conjugation strength of the molecules. They found that the conductivity exhibited an exponential decay versus the number of nonconjugated bonds in the linker molecule, suggesting that these molecules can be viewed as being serial connections of electrically insulating (nonconjugated) and conductive (conjugated) parts.

Selection of the binding groups connecting the molecules to the nanoparticle is also important for the electron transport. Chen et al. ${ }^{7}$ reported on the effect of the binding group on the single-molecule conductivity on an $\mathrm{Au}(111)$ surface using an Au scanning tunnel microscope tip. They found that the contact resistance, representing the strength of the electronic coupling between the molecules and the electrode, was very sensitive to the binding group, which decreased in the order: thiol $(-\mathrm{SH})>$ amine $\left(-\mathrm{NH}_{2}\right)>$ carboxylic acid $(-\mathrm{COOH})$, although the tunneling decay constant representing the efficiency of the electron transport was not dependent on the binding group.

In general, if a strong electronic coupling between a molecule and an electrode is realized, as in the case of Au-S bonds having a large polarization field at the metal-molecule 
interface, then the energy levels in the molecule broaden and the intrinsic electronic structure becomes ambiguous. ${ }^{8}$ If a molecule is adsorbed on a metal's surface with weak physisorption rather than strong chemisorption, the tunneling of electrons between the nanoparticles should be dominated by the intrinsic electronic structure of the molecule.

An assembly of gold nanoparticles protected by an electronically active conjugated molecule coupled with noncovalent bonding is an ideal system to study the effect of molecular structure and the frontier orbitals on the single electron conduction between gold nanoparticles, because it avoids a contribution from the binding groups, and reflects the contribution of the intrinsic electronic structure of the molecules and gold nanoparticles on the electron transport properties.

In this work, we have synthesized gold nanoparticles protected by a porphyrin derivative coupled via physisorption. meso-5,10,15,20-tetrakis(2-thienyl)porphyrin (2T) was selected for this purpose, because of its electronically active conjugated system, as well as its planar and robust structure. Gold nanoparticles protected by 2T (2T-AuNP) were assembled and the temperature-dependent electron transport properties were investigated. The 2T-AuNP assembly films showed a crossover around $50 \mathrm{~K}$ occurred from an Arrhenius-type thermally assisted hopping mechanism to an Efros-Shklovskii-type variable-range hopping (ES-VRH) mechanism with a strong Coulomb interaction regime.

\section{EXPERIMENTAL}

\section{A. Synthesis of 2T-AuNP}

The meso-5,10,15,20-tetrakis(2-thienyl)porphyrin used was synthesized using a method described in the literature. ${ }^{9}$ Preparation of the gold nanoparticles protected by 2T was carried out in a two-phase system, as described by Brust et $a l .{ }^{4} \mathrm{~A}$ volume of $70 \mathrm{ml}$ of an aqueous solution of $\mathrm{HAuCl}_{4}$ $3 \mathrm{H}_{2} \mathrm{O}(252 \mathrm{mg}, 0.64 \mathrm{mmol})$ was mixed with $210 \mathrm{ml}$ of a toluene solution of tetraoctylammonium bromide (TOAB). The two phases were stirred until all the tetrachloroaurate had transferred into the toluene phase. Then, $105 \mathrm{ml}$ of a toluene solution of $2 \mathrm{~T}$ (98 $\mathrm{mg}, 0.15 \mathrm{mmol})$ was added, and the mixture was stirred for a period of $15 \mathrm{~min}$. Finally, $10 \mathrm{ml}$ of an aqueous solution of $\mathrm{NaBH}_{4}(105 \mathrm{mg}, 2.77 \mathrm{mmol}$ ) was added slowly. After stirring for a further $20 \mathrm{~min}$, the toluene phase was separated and concentrated. Ethanol was then added, and the resulting precipitate was washed with ethanol and toluene to obtain gold nanoparticles protected by $2 \mathrm{~T}$ (2T-AuNP).

Elemental analysis of the 2T-AuNP showed C:H:N:S $=6.08 \%: 0.72 \%: 0.54 \%: 1.05 \%$, which corresponded to a ratio of 2T:gold atoms of 1:33. The cross-sectional area of $2 \mathrm{~T}$ for the flat and edge-on configurations was estimated from the molecular model compiled by the MERCURY and IMAGEJ (Ref. 10) [Fig. S1 (Ref. 11)]. Geometry optimization of 2T was carried out using density-functional theory calculations employing the B3LYP model with a 6-31G(d) basis set performed using the GAUSSIAN 03 software package. ${ }^{12}$

\section{B. Characterization of 2T-AuNP}

The average diameter of the gold nanoparticles was examined using transmission electron microscopy (TEM) and small-angle x-ray scattering (SAXS) in solution [see Fig. S2 (Ref. 11)]. TEM measurements were carried out using a Hitachi HD-2000 TEM employing an acceleration voltage of $200 \mathrm{kV}$. Samples for the TEM measurements were prepared on a carbon-coated $\mathrm{Cu}$ grid (Okenshoji Co. Ltd., Japan) by drop casting a toluene solution of the 2T-AuNP suspension, and then allowing the toluene to evaporate. The results were in good agreement with the SAXS data. The SAXS data were measured using a Rigaku Nanoviewer IP diffractometer employing $\mathrm{Cu} K \alpha$ radiation $(\lambda=1.5418 \AA)$ and a graphite monochromator $(40 \mathrm{kV}, 30 \mathrm{~mA})$. A $N, N$-dimethylformamide (DMF) solution of the gold nanoparticles was measured in the transmittance SAXS mode and the scattering profile was analyzed using the Rigaku GXRR software package. An average diameter of the 2T-AuNPs of $4.0 \mathrm{~nm}$ and a normalized variance of $10.3 \%$ were obtained using the least-squares method over a scanning range of 0.5 to $4.0^{\circ}$ using a structural factor of unity [Fig. S3 (Ref. 11)].

UV-vis-NIR, IR, and Raman spectroscopy data were obtained using a Perkin-Elmer Lambda-19 spectrophotometer, a Thermo scientific Nicolet 6700 spectrometer, and a Jasco RMP-210S microscope with an Ar-laser excitation source at $532 \mathrm{~nm}$, respectively. The synthesized 2T-AuNP samples were measured in a DMF solution to obtain the UV-vis-NIR data and $\mathrm{KBr}$ pellets for the IR and Raman data, respectively. The UV-vis-NIR data of the assembly films were measured on $\mathrm{SiO}_{2}$ substrates. The IR spectra consisted of 64 accumulations with a resolution of $4 \mathrm{~cm}^{-1}$ using an mercury cadmium telluride detector.

\section{Fabrication and transport properties of the 2T-AuNP assemblies}

$\mathrm{SiO}_{2}$ substrates (VIOSIL, KEN-SX) with a flatness of $<0.2 \mathrm{~nm}$ per $1 \mu \mathrm{m}^{2}$ were obtained from Shin-Etsu Chemical Co. Ltd., Japan. Gold electrodes with a thickness and a gap of $50 \mathrm{~nm}$ and $30 \mu \mathrm{m}$, respectively, and a width of $9 \mathrm{~mm}$ were deposited onto $\mathrm{SiO}_{2}$ substrates using vacuum evaporation. The $\mathrm{SiO}_{2}$ substrates coated with the gold electrodes were placed in a glass vial containing $50 \mathrm{ml}$ of a colloidal DMF solution of $2 \mathrm{~T}-\mathrm{AuNP}(0.01 \mathrm{mg} / \mathrm{ml})$. The solvent was evaporated at $40^{\circ} \mathrm{C}$ in a vacuum to fabricate the $2 \mathrm{~T}$-AuNP assembly films. Electrical contacts were formed using carbon paste and were used to attach $25 \mu \mathrm{m} \phi$ gold wires. Temperature-dependent $\mathrm{dc}$ resistance and current-voltage $(I-V)$ characteristics were measured using a homemade cryostat with a temperature control system over the range 3004.2 K. The current was monitored using a Keithley 6517 electrometer with a constant bias voltage in the range -5 to $+5 \mathrm{~V}$. Measurements were carried out on at least five samples to confirm the reproducibility. The relative dielectric constant of $2 \mathrm{~T}$ was measured using a two-probe ac impedance method at frequencies in the range 100-1 $000000 \mathrm{~Hz}$ (HP 4194A) at room temperature. The electrical contacts were prepared using gold paste (No. 8560, Tokuriki Chemical Research Co. Ltd., Japan) to attach $10 \mu \mathrm{m} \phi$ gold wires to the 2T pellet. Atomic force microscopy (AFM) images were obtained using a Seiko SPA 400 with an SPI 3800 probe station operating in the dynamic force mode. Commer- 


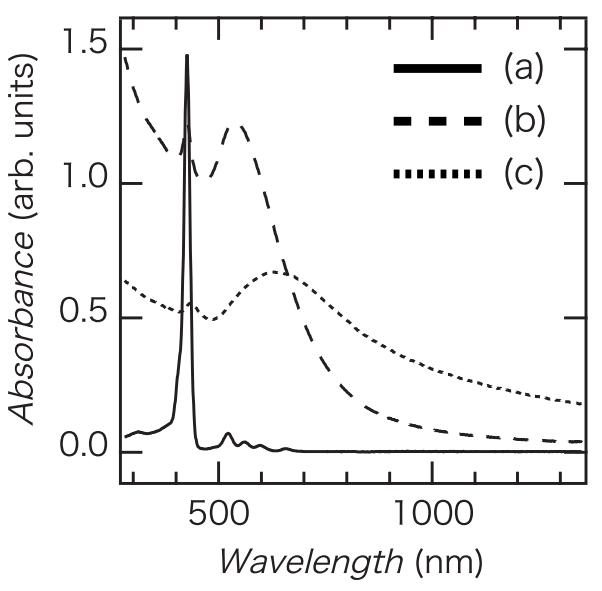

FIG. 1. UV-vis-NIR spectra of (a) 2T, (b) 2T-AuNP nanoparticles measured in a DMF solution, and (c) a 2T-AuNP assembly on an $\mathrm{SiO}_{2}$ substrate.

cially available $\mathrm{Si}$ cantilevers with a force constant of $20 \mathrm{~N} \mathrm{~m}^{-1}$ were used.

\section{RESULTS AND DISCUSSION}

\section{A. Characterization of 2T-AuNP}

A $4 \mathrm{~nm}$ diameter spherical gold nanoparticle contains approximately 1750 gold atoms ${ }^{13}$ and has a surface area of approximately $50 \mathrm{~nm}^{2}$. From elemental analysis, a single gold nanoparticle should adsorb approximately 50 molecules of $2 \mathrm{~T}$ (ratio of $2 \mathrm{~T}$ : gold atoms $=1: 33$ ). The cross-sectional area of a $2 \mathrm{~T}$ molecule that can be adsorbed on a $4 \mathrm{~nm}$ gold nanoparticle was estimated to be $1.73 \mathrm{~nm}^{2}$ and $0.78 \mathrm{~nm}^{2}$, respectively, for the flat and edge-on configurations [see Fig. S4 (Ref. 11)]. These corresponded to 29 and 64 molecules, respectively. The 50 molecules adsorbed on a $4 \mathrm{~nm}$ gold nanoparticle implies that the $2 \mathrm{~T}$ molecules were adsorbed with a tilted conformation on the gold surface.

\section{B. Adsorption mechanism between 2T and gold nanoparticles}

The interaction between gold nanoparticles and $2 \mathrm{~T}$ on adsorption was examined. The 2T-AuNP exhibited Soret bands in UV-vis-NIR spectrum originating from the 2T [Fig. 1(a)] and plasmon bands of the gold nanoparticles occurring at $426 \mathrm{~nm}$ and $539 \mathrm{~nm}$, respectively [Fig. 1(b)]. The Q band, corresponding to the highest occupied molecular orbitallowest unoccupied molecular orbital transition of 2T, was not observed due to the strong plasmon band. The 2T Soret band did not change after fabrication of a 2T-AuNP indicating that no distortion of the macrocycle ring (redshift), ${ }^{14} \mathrm{~J}$ or $\mathrm{H}$ aggregation (redshift or blueshift), ${ }^{15}$ charge transfer (redshift), ${ }^{16}$ or protonation (redshift) (Ref. 17) of the $2 \mathrm{~T}$ occurred on the gold surface. The electronic structure of $2 \mathrm{~T}$ was maintained on formation of the 2T-AuNP. The plasmon band of the gold nanoparticles did not change up to a period of $4 \mathrm{~h}$ [Fig. S5 (Ref. 11)], showing the stability of the 2T-AuNP. ${ }^{18}$ All the ${ }^{1} \mathrm{H}$-NMR peaks of 2T-AuNP were significantly broadened [Fig. S6 (Ref. 11)], indicating that the $2 \mathrm{~T}$ molecules were closely bound to the gold surface. ${ }^{19}$
The adsorption mechanism between the $2 \mathrm{~T}$ and gold nanoparticles was investigated to ascertain whether it had a covalent or noncovalent nature. Ohta et al. ${ }^{20}$ reported the possibility of a cleavage of the C-S bond, resulting in an $\mathrm{Au}-\mathrm{S}$ bond when thiophene is adsorbed on a gold surface. We measured the IR and Raman spectra to confirm the molecular structure of 2T on the gold nanoparticles [see Fig. S7 (Ref. 11)]. The IR spectra showed the C-H stretching mode of thiophene (3098 and $3062 \mathrm{~cm}^{-1}$ ) (Ref. 21) without the alkyl C-H stretching mode of butane thiol (occurring around $2900 \mathrm{~cm}^{-1}$ ) expected to be formed by cleavage of the C-S bond of the thiophene ring. Since neither the IR nor the Raman spectra showed any change on adsorption, the adsorption of $2 \mathrm{~T}$ on the gold nanoparticles was assumed to have a noncovalent bonding nature. Therefore, we propose that the gold nanoparticles can be protected by $2 \mathrm{~T}$ via physisorption.

\section{Fabrication of a 2T-AuNP assembly}

Figure 1(c) shows the UV-vis-NIR transmission spectrum of a 2T-AuNP assembly fabricated on an $\mathrm{SiO}_{2}$ substrate. The Soret and plasmon bands were shifted to the red (from 426 $\mathrm{nm}$ and $540 \mathrm{~nm}$ to $436 \mathrm{~nm}$ and $643 \mathrm{~nm}$, respectively) and were broadened owing to nanoparticle aggregation. ${ }^{22}$ The IR spectra [Fig. S7 (Ref. 11)] showed C-H stretching mode of thiophene (3098 and $3062 \mathrm{~cm}^{-1}$ ) without alkyl C-H stretching mode of TOAB (at around $2900 \mathrm{~cm}^{-1}$ ), indicating TOAB molecules did not exist in 2T-AuNP, although Schiffrin et $a l .^{23}$ have pointed out the difficulty of removing TOAB from AuNPs. AFM images of a 2T-AuNP assembly between the electrode gap were obtained after charge transport measurements, as shown in Fig. 2. The gold nanoparticles were confirmed to have a rounded shape and an aggregated structure was formed between electrodes. The observed particle diameter $(\sim 100 \mathrm{~nm})$ was significantly larger than the estimated value from the TEM and SAXS measurement because the radius of curvature of the cantilevers (approximately $10 \mathrm{~nm}$ ) was much larger than the particle size (approximately $4 \mathrm{~nm}$ ). The height of a single 2TAuNP assembly was confirmed to be about $5 \mathrm{~nm}$ [Fig. S8 (Ref. 11)], suggesting that the particle size was maintained in the 2T-AuNP aggregated structure. The height of the network was greater than $50 \mathrm{~nm}$, the electrode height, indicating that the 2T-AuNP assembly had a three-dimensional structure.

\section{Temperature dependence of the charge transport of a 2T-AuNP assembly structure}

Figure 3 shows temperature-dependent normalized resistance of an assembly structure of 2T-AuNP. The resistivity showed a semiconducting behavior from room temperature to $50 \mathrm{~K}$ and was described by an Arrhenius plot $\left(T^{-1}\right)$ with the activation energy of $15 \mathrm{meV}$. This magnitude is of the same order as the assemblies reported previously with similar particle diameters. ${ }^{24}$ Ohmic $I-V$ characteristics were also confirmed at each measurement point [Fig. S9 (Ref. 11)]. Thermal activated hopping dominated the temperature range between 296 and $50 \mathrm{~K}$. On the other hand, the resistivity below $50 \mathrm{~K}$ showed a deviation from the $T^{-1}$ plot. So far, 


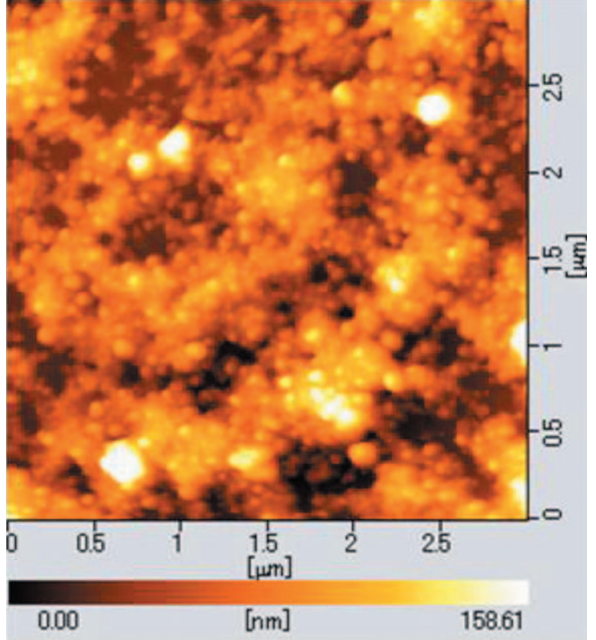

FIG. 2. (Color) AFM images of a 2T-AuNP assembly obtained within the electrode gap after charge transport measurements.

semiconducting charge transport behavior in nanoparticle assemblies has been reported to often obey the VRH model,

$$
R \propto \exp \left[\left(\frac{T_{0}}{T}\right)^{\gamma}\right],
$$

where $T_{0}$ is a constant and the value of $\gamma$ depends on the dimensionality. If strong Coulomb interactions exist, then the system obeys a $\gamma=1 / 2$ law, which is independent of the dimensionality, and is known as ES-VRH. ${ }^{25,26}$ The $T^{-1 / 2}$ plot for a 2T-AuNP assembly is shown in Fig. 3, and indicates a linear relationship existed below $50 \mathrm{~K}$, with a kink in the graph occurring around $10 \mathrm{~K}$.

Below $10 \mathrm{~K}$, the behavior again deviated from the ESVRH behavior. The $I-V$ characteristics were measured at 4.2 $\mathrm{K}$ to elucidate the transport mechanism below $10 \mathrm{~K}$. The $I-V$ characteristics at $4.2 \mathrm{~K}$ showed a threshold behavior (Fig. 4). This behavior can be explained by the model of Middleton and Wingreen (the MW model), ${ }^{27}$ which is based on an electron transfer through the nanoparticle assembly taking into account randomly charged nanoparticles separated by tunnel junctions. The $I-V$ characteristics of this model are

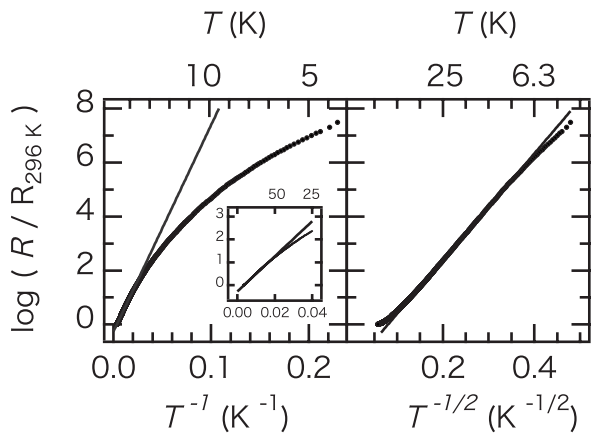

FIG. 3. Normalized resistivity of a 2T-AuNP assembly plotted vs $T^{-1}$ and $T^{-1 / 2}$. The solid lines denote linear fits in the temperature range $265-50 \mathrm{~K}$ (left) and 50-10 $\mathrm{K}$ (right).

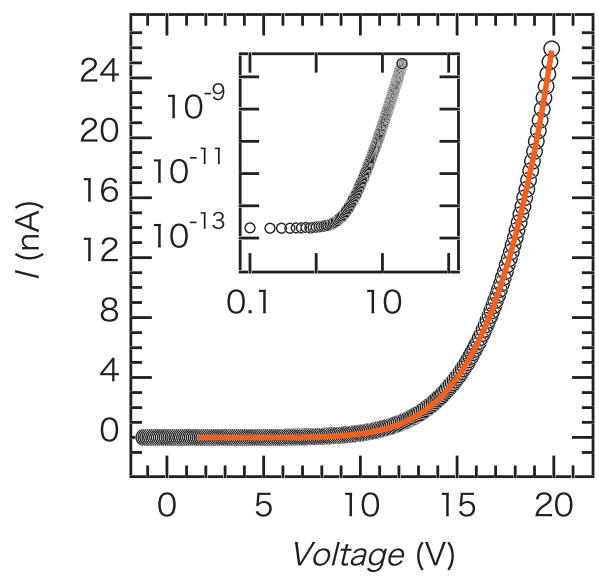

FIG. 4. (Color) $I$ - $V$ characteristics of a $2 \mathrm{~T}-\mathrm{AuNP}$ assembly at 4.2 $\mathrm{K}$. The red line denotes the MW model given by Eq. (2). The parameters $V_{T}$ and $\xi$ were $1.6 \mathrm{~V}$ and $6.0 \mathrm{~V}$, respectively. The inset shows the log-log plots.

$$
I \propto\left(V / V_{T}-1\right)^{\xi},
$$

where $V_{T}$ is the Coulomb-blockade threshold voltage and $\xi$ is the dimensionality of the current pathway, which depends on the structure. The values of $\xi=1$ and 5/3 were calculated for infinite one-dimensional and two-dimensional arrays, respectively, and numerical studies indicated that $2.2<\xi<3.0$ for multilayered arrays. ${ }^{28}$ The model reproduced the $I-V$ characteristics at $4.2 \mathrm{~K}$ well, indicating that electrons can tunnel through the nanoparticles affected by the Coulomb blockade, although the parameter $\xi$ was substantially larger than the theoretical value.

\section{E. Electron transport mechanism}

The application of the ES-VRH model to granular systems sometimes results in a hopping distance $\left(r_{h o p}\right)$ with physically unreasonable values, i.e., hundreds of nanometer or larger, ${ }^{29}$ which cannot explain how the Coulomb interactions between the initial and final nanoparticles correlate. To confirm the applicability of the ES-VRH model, ${ }^{25}$ we estimated the values of localization length $(a)$ (Refs. 26, 30, and 31 ) and the $r_{\text {hop }}$ (Refs. 29 and 30) of the conduction electrons from

$$
\begin{gathered}
T_{0}=\beta \frac{e^{2}}{4 \pi \varepsilon_{r} \varepsilon_{0} k_{B} a}, \\
\kappa=\frac{1}{a}, \\
r_{\text {hop }}=\frac{1}{4 \kappa}\left(\frac{T_{0}}{T}\right)^{1 / 2},
\end{gathered}
$$

where $\kappa$ is the tunneling decay constant, $k_{B}$ is the Boltzmann constant, $e$ is the electron charge, $\varepsilon_{0}$ is the dielectric constant in a vacuum, and $\varepsilon_{r}$ is the relative dielectric constant of the surrounding medium. The coefficient $\beta$ depends on the dimensionality (for two dimensions $\sim 6.5$, and for three dimensions $\sim 2.8) .^{32}$ 
We assumed that a three-dimensional current pathway was realized due to the structural features seen in the AFM images (Fig. 2). From Eqs. (1) and (3), we obtained $a$ $=1.5 \mathrm{~nm}\left(T=50-10 \mathrm{~K}, T_{0}=2077 \mathrm{~K}\right.$, and $\varepsilon_{r}=15$ obtained from dielectric measurements on 2T). The parameter $a$ describes the decay length of the electronic wave functions. In nanoparticle arrays, the magnitude of $a$ is equivalent to the size of each nanoparticle, ${ }^{27,33}$ and larger values imply stronger coupling of the nanoparticles. ${ }^{31}$ In the temperature range 50-10 K, the value of $r_{\text {hop }}$ increased with decreasing temperature, from 2.4 to $5.4 \mathrm{~nm}$. These distances were relatively small and are physically reasonable when compared with those of earlier reports. ${ }^{29}$ Because the 2T-AuNP assemblies contained nanoparticles with a diameter of approximately 4 $\mathrm{nm}$, the increase in the value of $r_{\text {hop }}$ from a value of $2.4 \mathrm{~nm}$ represents the crossover point of the charge transport mechanism from an Arrhenius-type thermal-activated hopping (i.e., nearest-neighbor hopping) to an ES-VRH-type hopping mechanism around $50 \mathrm{~K}$. The localization length of $a$ $=1.5 \mathrm{~nm}$ indicates that a single electron could be localized within a single nanoparticle and that a single gold nanoparticle in a 2T-AuNP assembly could be in the charged state in the regime of the so-called single-electron charging effect, ${ }^{34,35}$ i.e., below $50 \mathrm{~K}$. It is worth noting that, as shown in Fig. 2, the 2T-AuNP were assembled in the form of a continuous multilayer film with many empty spaces between the relatively large electrodes gap $(30 \mu \mathrm{m})$ compared to the other reports in which closely packed nanoparticle monolayer or multilayer with no empty spaces were created between the gap of 500-600 nm (Ref. 31) and 2-5 $\mu \mathrm{m} .{ }^{26}$ The physically reasonable estimated values of localization length $a$ and the $r_{h o p}$ suggests appropriateness of ES-VRH-type hopping mechanism in 2T-Au assemblies.

The Coulomb-blockade behavior observed in the $I-V$ characteristics at $4.2 \mathrm{~K}$ implies that single-electron tunneling occurred below $10 \mathrm{~K}$. Single-electron tunneling is essentially independent of temperature because it can be realized when the charging energy can overcome the thermal energy. ${ }^{34}$ The reason why the $T^{-1 / 2}$ dependence decreases below $10 \mathrm{~K}$, as shown in Fig. 3, is because of the contribution of the Coulomb blockade. The large value of $\xi$ obtained in the $I-V$ characteristics at $4.2 \mathrm{~K}$ strongly suggests a contribution of the ES-VRH-type hopping, although further study will be required.

Some studies have explained the $T^{-1 / 2}$ behavior using a coherent tunneling model or a random resistance model. The coherent tunneling model was proposed to explain longrange hopping in nanoparticle assemblies. ${ }^{31}$ In this model, an electron tunnels into a nanoparticle and another electron escapes from the same nanoparticle at the same time. Coherent tunneling provides a conduction channel at low applied bias through several junctions, where otherwise the Coulomb blockade would suppress any current flow. ${ }^{36}$ This behavior is observed in a system with covalent bonds between the gold nanoparticles and an organic molecule, such as a saturated ${ }^{31}$ or conjugated molecule. ${ }^{3}$ Coherent tunneling is characterized by a power-law $I-V$ behavior in the Coulomb-blockade regime, although only a strong current suppression below $V_{T}$ $(1.6 \mathrm{~V})$ was observed in the present system. In the case of a random resistance model, where $T^{-1 / 2}$ behavior was the result of a percolation pathway with least resistance, the resistivity behavior only depends on its geometry. ${ }^{37}$ If the random resistance model dominates, then the temperature dependence should follow a $T^{-1}$ or $T^{-1 / 2}$ behavior over the entire temperature range. The resistivity of a 2T-AuNP assembly having a crossover from $T^{-1}$ to $T^{-1 / 2}$ behavior around $50 \mathrm{~K}$ cannot be explained using the random resistance model.

\section{CONCLUSIONS}

We have synthesized gold nanoparticles with an average diameter of $4.0 \mathrm{~nm}$ protected by physisorbed $2 \mathrm{~T}$, which were fabricated into an assembly structure of 2T-AuNP. We fabricated assemblies of gold nanoparticles protected by a $\pi$-conjugated molecules via noncovalent bonding and measured their electronic transport properties. On lowering the temperature, the temperature-dependent resistivity of a $2 \mathrm{~T}$ AuNP assembly structure showed a crossover from an Arrhenius-type thermal-activated hopping to an ES-VRHtype hopping mechanism around $50 \mathrm{~K}$. In addition, a Coulomb blockade influenced the strong Coulomb repulsion between the nanoparticles below $10 \mathrm{~K}$. The existence of a crossover is supported by the hopping distance of $2.4 \mathrm{~nm}$ at $50 \mathrm{~K}$, which implies that the hopping distance between nearest-neighbor particles increases to $5.4 \mathrm{~nm}$ at $10 \mathrm{~K}$. The localization length of $1.5 \mathrm{~nm}$ estimated from the ES-VRH model was smaller than the 2T-AuNP diameter, indicating a weak coupling between NP units and the single-electron charging effect. Below $10 \mathrm{~K}$, the Coulomb-blockade behavior caused a smaller temperature dependence of the resistivity. The appearance of the ES-VRH behavior and the Coulomb-blockade behavior indicated that the $2 \mathrm{~T}$ and gold nanoparticles in the 2T-AuNP assembly were electrically isolated from each other, although the gold nanoparticles were surrounded by an electrically active organic molecule. This was due to the weak adsorption of $2 \mathrm{~T}$ on the gold nanoparticles via physisorption.

\section{ACKNOWLEDGMENTS}

This work was supported in part by a Grant-in-Aid for Science Research from the Ministry of Education, Culture, Sports, Science, and Technology of Japan. Y.N. acknowledges Japan Society for the Promotion of Science (JSPS). 
${ }^{1}$ A. Zabet-Khosousi, P. E. Trudeau, Y. Suganuma, A. A. Dhirani, and B. Statt, Phys. Rev. Lett. 96, 156403 (2006); J. B. Pełka, M. Brust, P. Gierłowski, W. Paszkowicz, and N. Schell, Appl. Phys. Lett. 89, 063110 (2006).

${ }^{2}$ H. Nakanishi, K. J. M. Bishop, B. Kowalczyk, A. Nitzan, E. A. Weiss, K. V. Tretiakov, M. M. Apodaca, R. Klajn, J. F. Stoddart, and B. A. Grzybowski, Nature (London) 460, 371 (2009); X. N. Xie, Y. Xie, X. Gao, C. H. Sow, and A. T. S. Wee, Adv. Mater. 21, 3016 (2009).

${ }^{3}$ M. Minamoto, M. M. Matsushita, and T. Sugawara, Polyhedron 24, 2263 (2005); P. Nickels, M. M. Matsushita, M. Minamoto, S. Komiyama, and T. Sugawara, Small 4, 471 (2008); T. Sugawara, M. Minamoto, M. M. Matsushita, P. Nickels, and S. Komiyama, Phys. Rev. B 77, 235316 (2008).

${ }^{4}$ M. Brust, M. Walker, D. Bethell, D. J. Schiffrin, and R. Whyman, J. Chem. Soc., Chem. Commun. 1994, 801; M. Brust, J. Fink, D. Bethell, D. J. Schiffrin, and C. Kiely, ibid. 1995, 1655.

${ }^{5}$ V. Torma, O. Vidoni, U. Simon, and G. Schmid, Eur. J. Inorg. Chem. 2003, 1121 (2003).

${ }^{6}$ J. M. Wessels, H.-G. Nothofer, W. E. Ford, F. von Wrochem, F. Scholz, T. Vossmeyer, A. Schroedter, H. Weller, and A. Yasuda, J. Am. Chem. Soc. 126, 3349 (2004).

${ }^{7}$ F. Chen, X. Li, J. Hihath, Z. Huang, and N. Tao, J. Am. Chem. Soc. 128, 15874 (2006).

${ }^{8}$ N. J. Tao, Nat. Nanotechnol. 1, 173 (2006).

${ }^{9}$ A. D. Adler, F. R. Longo, J. D. Finarelli, J. Goldmacher, J. Assour, and L. Korsakoff, J. Org. Chem. 32, 476 (1967).

${ }^{10}$ MERCURY Version 2.3, The Cambridge Crystallographic Data Centre, UK., IMAGEJ Version $1.43 \mathrm{u}$, The National Institutes of Health, USA.

${ }^{11}$ See supplementary material at http://link.aps.org/supplemental/ 10.1103/PhysRevB.82.205420 for supporting figures.

${ }^{12}$ M. J. Frisch et al., Gaussian, Inc., Wallingford, CT, 2004.

${ }^{13}$ G. Schmid, Chem. Rev. 92, 1709 (1992).

${ }^{14}$ J. P. Hill, I. J. Hewitt, C. E. Anson, A. K. Powell, A. L. McCarty, P. A. Karr, M. E. Zandler, and F. D’Souza, J. Org. Chem. 69, 5861 (2004).

${ }^{15}$ M. Zhou, S. Ouyang, Z. Liu, G. Lu, S. Gao, and Z. Li, Vib. Spectrosc. 49, 7 (2009); M. Gouterman, D. Holten, and E. Lieberman, Chem. Phys. 25, 139 (1977); J. Zimmermann, U. Siggel, J.-H. Fuhrhop, and B. Roder, J. Phys. Chem. B 107, 6019 (2003); K. A. Scherz and W. W. Parson, Biochim. Biophys. Acta 766, 653 (1984).

${ }^{16}$ A. Hosseini, S. Taylor, G. Accorsi, N. Armaroli, C. A. Reed, and P. D. W. Boyd, J. Am. Chem. Soc. 128, 15903 (2006).

${ }^{17}$ I. Gupta and M. Ravikanth, J. Photochem. Photobiol., A 177, 156 (2006).

${ }^{18}$ S. K. Ghosh and T. Pal, Chem. Rev. 107, 4797 (2007); M. M. Maye, J. Luo, I-I. S. Lim, L. Han, N. N. Kariuki, D. Rabinovich, T. Liu, and C.-J. Zhong, J. Am. Chem. Soc. 125, 9906 (2003); J. A. Creighton, C. G. Blatchford, and M. G. Albrecht, J. Chem. Soc., Faraday Trans. 2 75, 790 (1979); K. U. von Raben, R. K. Chang, and B. L. Laube, Chem. Phys. Lett. 79, 465 (1981); S. Link and M. A. El-Sayed, J. Phys. Chem. B 103, 8410 (1999).

${ }^{19}$ C. Mayer, Annu. Rep. NMR Spectrosc. 55, 205 (2005).

${ }^{20}$ E. O. Sako, H. Kondoh, I. Nakai, A. Nambu, T. Nakamura, and
T. Ohta, Chem. Phys. Lett. 413, 267 (2005).

${ }^{21}$ T. Matsuura and Y. Shimoyama, Eur. Phys. J. E 7, 233 (2002).

${ }^{22}$ A. M. Kalsin, A. O. Pinchuk, S. K. Smoukov, M. Paszewski, G. C. Schatz, and B. A. Grzybowski, Nano Lett. 6, 1896 (2006); A. O. Pinchuk, A. M. Kalsin, B. Kowalczyk, G. C. Schatz, and B. A. Grzybowski, J. Phys. Chem. C 111, 11816 (2007).

${ }^{23}$ C. A. Waters, A. J. Mills, K. A. Johnson, and D. J. Schiffrin, Chem. Commun. (Cambridge) 2003, 540.

${ }^{24}$ For example, $38 \mathrm{meV}$ for $4.6-6.5 \mathrm{~nm}$ diameter, R. Parthasarathy, X. M. Lin, K. Elteto, T. F. Rosenbaum, and H. M. Jaeger, Phys. Rev. Lett. 92, 076801 (2004); $20 \mathrm{meV}$ for $6 \mathrm{~nm}$ diameter, M. Brust, D. Bethell, C. J. Kiely, and D. J. Schiffrin, Langmuir 14, 5425 (1998); 47-58 meV for $4 \mathrm{~nm}$ diameter, Y. Joseph, I. Besnard, M. Rosenberger, B. Guse, H. G. Nothofer, J. M. Wessels, U. Wild, A. Knop-Gericke, D. Su, R. Schlögl, A. Yasuda, and T. Vossmeyer, J. Phys. Chem. B 107, 7406 (2003).

${ }^{25}$ A. L. Efros and B. I. Shklovskii, J. Phys. C 8, L49 (1975); B. I. Shklovskii and A. L. Efros, Electronic Properties of Doped Semiconductors (Springer-Verlag, Berlin, 1988).

${ }^{26}$ D. Yu, C. Wang, B. L. Wehrenberg, and P. Guyot-Sionnest, Phys. Rev. Lett. 92, 216802 (2004); B. L. Wehrenberg, D. Yu, J. Ma, and P. Guyot-Sionnest, J. Phys. Chem. B 109, 20192 (2005); H. E. Romero and M. Drndic, Phys. Rev. Lett. 95, 156801 (2005).

${ }^{27}$ A. A. Middleton and S. N. Wingreen, Phys. Rev. Lett. 71, 3198 (1993).

${ }^{28}$ C. T. Black, C. B. Murray, R. L. Sandstrom, and S. Sun, Science 290, 1131 (2000); H. Fan, K. Yang, D. M. Boye, T. Sigmon, K. J. Malloy, H. Xu, G. P. López, and C. Jeffrey Brinker, ibid. 304, 567 (2004).

${ }^{29}$ J. L. Dunford, Y. Suganuma, A.-A. Dhirani, and B. Statt, Phys. Rev. B 72, 075441 (2005); T. Dadosh, Y. Gordin, R. Krahne, I. Khivrich, D. Mahalu, V. Frydman, J. Sperling, A. Yacoby, and I. Bar-Joseph, Nature (London) 436, 677 (2005); Z.-M. Liao, J. Xun, and D.-P. Yu, Phys. Lett. A 345, 386 (2005); M. A. Rafiq, Y. Tsuchiya, H. Mizuta, S. Oda, S. Uno, Z. A. K. Durrani, and W. I. Milne, J. Appl. Phys. 100, 014303 (2006).

${ }^{30}$ A. Zabet-Khosousi and A. A. Dhirani, Chem. Rev. 108, 4072 (2008).

${ }^{31}$ T. B. Tran, I. S. Beloborodov, X. M. Lin, T. P. Bigioni, V. M. Vinokur, and H. M. Jaeger, Phys. Rev. Lett. 95, 076806 (2005); D. V. Averin and Y. V. Nazarov, ibid. 65, 2446 (1990).

${ }^{32}$ K. Xu, L. Qin, and J. R. Heath, Nat. Nanotechnol. 4, 368 (2009); V. Duc Nguyen, V. Lien Nguyen, and D. Toi Dang, Phys. Lett. A 349, 404 (2006).

${ }^{33}$ K. C. Beverly, J. F. Sampaio, and J. R. Heath, J. Phys. Chem. B 106, 2131 (2002).

${ }^{34}$ M. Amman, R. Wilkins, E. Ben-Jacob, P. D. Maker, and R. C. Jaklevic, Phys. Rev. B 43, 1146 (1991).

${ }^{35}$ B. Abeles, P. Sheng, M. D. Coutts, and Y. Arie, Adv. Phys. 24, 407 (1975).

${ }^{36}$ I. S. Beloborodov, A. V. Lopatin, and V. M. Vinokur, Phys. Rev. B 72, 125121 (2005).

${ }^{37}$ V. Ambegaokar, B. I. Halperin, and J. S. Langer, Phys. Rev. B 4, 2612 (1971); K.-H. Müller, J. Herrmann, B. Raguse, G. Baxter, and T. Reda, ibid. 66, 075417 (2002); K.-H. Müller, G. Wei, B. Raguse, and J. Myers, ibid. 68, 155407 (2003). 